Tropical Journal of Pharmaceutical Research September 2014; 13 (9): 1527-1536

ISSN: $1596-5996$ (print); 1596-9827 (electronic)

(c) Pharmacotherapy Group, Faculty of Pharmacy, University of Benin, Benin City, 300001 Nigeria.

All rights reserved.

Available online at http://www.tjpr.org

Original Research Article

http://dx.doi.org/10.4314/tjpr.v13i9.21

\title{
Alternative Medicines for HIV/AIDS in Resource-Poor Settings: Insight from Traditional Medicines Use in Sub- Saharan Africa
}

\author{
Alfred Maroyi \\ Medicinal Plants and Economic Development (MPED) Research Centre; Department of Botany, University of Fort Hare, Private \\ Bag X1314, Alice 5700, South Africa \\ *For correspondence: Email: amaroyi@ufh.ac.za; Tel: +27406022320, Fax: +27866177642
}

Received: 26 February 2014

Revised accepted: 19 July 2014

\begin{abstract}
Purpose: To document the utilization of traditional medicines in managing human immunodeficiency virus/acquired immunodeficiency syndrome (HIVIAIDS) opportunistic infections in sub-Saharan Africa. Methods: This study is based on a review of literature published in scientific journals, books, reports from national, regional and international organizations, theses and conference papers obtained from libraries and electronic search of Google Scholar, ISI Web of Science, MEDLINE, Pubmed, Scopus and Science Direct.

Results: A total of 79 medical conditions related to HIVIAIDS were treated using 74 plant species. The common diseases treated by herbal remedies were bacterial/fungal infections, boosting of appetite/immunity, cold/cough, cryptococcal meningitis, diarrhea, fever, herpes simplex/zoster, oral/oesopharyngeal candidiasis, skin infections/rash, tuberculosis and wounds. More than threequarters of the documented plant species (63 species, $85.1 \%$ ) have anti-HIV active compounds.

Conclusion: This study reveals that traditional medicines are often used as alternative sources of medicines for HIVIAIDS opportunistic infections in sub-Saharan Africa. Further investigations are needed to explore the bioactive compounds of these herbal medicines, aimed at exploring the bioactive compounds that can be developed into anti-HIV drugs.
\end{abstract}

Keywords: Antiretroviral, HIVIAIDS, Sub-Saharan Africa, Traditional medicines

Tropical Journal of Pharmaceutical Research is indexed by Science Citation Index (SciSearch), Scopus, International Pharmaceutical Abstract, Chemical Abstracts, Embase, Index Copernicus, EBSCO, African Index Medicus, JournalSeek, Journal Citation Reports/Science Edition, Directory of Open Access Journals (DOAJ), African Journal Online, Bioline International, Open-J-Gate and Pharmacy Abstracts

\section{INTRODUCTION}

The acquired immunodeficiency syndrome (AIDS), caused by infection with the human immunodeficiency virus (HIV) has become a devastating epidemic in sub-Saharan Africa [1]. Ethnobotanical studies conducted in the following sub-Saharan African countries indicated that traditional healers and local communities extensively use medicinal plants to manage the effects of HIV/AIDS in Cameroon [2], Namibia
[3,4], Rwanda [5], South Africa [6-8], Sudan [9], Tanzania $[1,10]$ and Uganda [11-13].

Despite the increasing acceptance of traditional medicines as an additional and alternative therapeutic strategy in the fight against HIV/AIDS opportunistic diseases in sub-Saharan Africa [1$4,7,8,10-13]$, this rich indigenous knowledge on traditional remedies is not adequately documented. Documentation of plants used for treating and managing HIV/AIDS opportunistic diseases in sub-Saharan Africa is urgent as there 
is need to search for novel anti-HIV compounds. Moreover, an estimated $25 \%$ of pharmaceutical drugs and $11 \%$ of drugs considered essential by $\mathrm{WHO}$ are derived from plants; a large number of synthetic drugs obtained from precursor compounds originating from plants [14].

Medicinal plants used for treating and managing HIV/AIDS opportunistic diseases in sub-Saharan Africa are an integral part of a culturally accepted and holistic health care system that has been in existence for several generations. Therefore, traditional medicines are possible sources of complementary and alternative medicines for HIV/AIDS opportunistic diseases.

This review briefly examines the role of herbal medicines in the fight against HIV/AIDS opportunistic diseases in sub-Saharan Africa. It is hoped that this review will provide important and relevant information that will form a basis for further research to identify and isolate bioactive constituents that can be developed as drugs for the treatment and management of HIV/AIDS opportunistic diseases.

\section{EXPERIMENTAL}

In this study, the focus was on herbal plants that are used to treat or manage HIV/AIDS opportunistic diseases in sub-Saharan Africa, and also reported to have anti-viral bioactivity based on in vitro or in vivo studies. Only papers that included information on plant identity, the HIV/AIDS opportunistic diseases treated and plant part(s) used were included in this review.

The International Plant Name Index (www.ipni.org) and the Royal Botanic Garden and Missouri Botanic Garden plant name database (www.theplantlist.org) were used to validate plant scientific names, plant families and plant authorities. In addition to this, the anti-viral efficacy of the documented plant species, either in vitro or in vivo was also verified from published literature. Therefore, this review draws heavily on the results of ethnobotanical surveys undertaken in contemporary sub-Saharan Africa on traditional medicines used to treat or manage HIV/AIDS opportunistic diseases [1-13], as well as secondary data collected through a review of 60 research reports, policy documents, reports from national, regional and international organizations, and conference papers published in scientific journals (53), books (4), theses (2) and one as an abstract.
Literature was searched on international online databases such as ISI Web of Science, MEDLINE, Science Direct, Scopus and Google Scholar using specific search terms such as "herbal medicines used to treat HIV/AIDS opportunistic diseases", "medicinal plants used to treat HIV/AIDS opportunistic diseases", "traditional medicines used to treat HIV/AIDS opportunistic diseases", and "anti-viral activity of [each documented plant species]". References were also identified by searching the library collections of the National Herbarium and Botanic Gardens, Zimbabwe and University of Fort Hare, South Africa.

\section{FINDINGS}

This study recorded 74 plant species widely used traditionally in treating and managing HIV/AIDS opportunistic diseases in sub-Saharan Africa (Table 1. These medicinal plants were distributed among 37 families and 65 genera. The majority of medicinal plants $(50,67.6 \%)$ used to treat and manage HIV/AIDS opportunistic diseases in subSaharan Africa were from 13 families (Table 2). Plant families with the highest number of medicinal plants were Fabaceae sensu lato (11 species), followed by Asteraceae and Euphorbiaceae (5 species each), Combretaceae, Lamiaceae and Myrtaceae (4 species each), Amaryllidaceae, Cucurbitaceae and Phyllanthaceae (3 species each), Apiaceae, Celastraceae, Clusiaceae and Xanthorrhoeaceae (2 species each). The rest of the families were represented by one species each (Table 1). The genera with highest number of species were Combretum with three species, followed by Artemisia, Cucurbita, Euphorbia, Garcinia, Plectranthus, Senna and Syzygium with 2 species each (Table 1).

\section{Growth form and plant parts used}

Trees (43.2\%) were the primary sources of the medicinal plants used for treating and managing HIV/AIDS opportunistic diseases in sub-Saharan Africa, followed by shrubs (27\%), herbs (24.3\%) and climbers $(5.4 \%)$ (Table 1). The plant parts used for making herbal preparations were the bark, bulbs, fruits, leaves, leaf sap, roots, stem, tubers and twigs (Table 1 ). The leaves $(67.6 \%)$ were the most frequently used, followed by roots $(35.1 \%)$, bark (31.1\%), bulb and seeds $(5,4 \%$ each), fruits, stem and twigs ( $2.7 \%$ each), tubers and leaf sap (1.4\% each) (Table 1). 
Table 1: Plant species used to treat and manage various HIV/AIDS opportunistic diseases in sub-Saharan Africa

\begin{tabular}{|c|c|c|c|c|}
\hline $\begin{array}{l}\text { Family, species } \\
\text { name }\end{array}$ & $\begin{array}{l}\text { Growth } \\
\text { habit }\end{array}$ & $\begin{array}{l}\text { Part(s) } \\
\text { used }\end{array}$ & $\begin{array}{l}\text { Condition treated, country and references } \\
\text { in brackets }\end{array}$ & $\begin{array}{l}\text { Pharmacological } \\
\text { activities }\end{array}$ \\
\hline \multicolumn{5}{|l|}{ Amaryllidaceae } \\
\hline $\begin{array}{l}\text { Allium sativum } \\
\mathrm{L} \text {. }\end{array}$ & Herb & Bulb & $\begin{array}{l}\text { Aspergillosis, oesopharyngeal candidiasis } \\
\text { (South Africa) [8], skin infections (Cameroon) } \\
\text { [2] }\end{array}$ & $\begin{array}{l}\text { HIV-1 reverse } \\
\text { transcriptase } \\
\text { inhibitory effect [15] }\end{array}$ \\
\hline $\begin{array}{l}\text { Crinum } \\
\text { macowani Baker }\end{array}$ & Herb & Bulb & $\begin{array}{l}\text { Acne, backache, blood cleansing, boils, cold, } \\
\text { fever, glandular swelling, kidney and bladder } \\
\text { diseases, rheumatism, wounds, tonic, } \\
\text { tuberculosis, venereal diseases (South } \\
\text { Africa) }[8,16,17]\end{array}$ & Anti-HIV activity [16] \\
\hline $\begin{array}{l}\text { Tulbaghia } \\
\text { violacea Harv. }\end{array}$ & Herb & Leaves & $\begin{array}{l}\text { Arthritis, asthma, cancer, cold, cough, } \\
\text { cryptococcal meningitis, fever, intestinal } \\
\text { worms, rheumatism, tuberculosis (South } \\
\text { Africa) }[8,16,17]\end{array}$ & Anti-HIV activity [16] \\
\hline $\begin{array}{l}\text { Anacardiaceae } \\
\text { Mangifera indica } \\
\text { L. } \\
\text { Apiaceae }\end{array}$ & Tree & $\begin{array}{l}\text { Bark/leave } \\
\text { s }\end{array}$ & $\begin{array}{l}\text { Cough, diarrhea, tuberculosis (Tanzania, } \\
\text { Uganda) }[1,11-13]\end{array}$ & $\begin{array}{l}\text { Inhibits HSV-1 and } \\
2 \text { replication [18] }\end{array}$ \\
\hline $\begin{array}{l}\text { Alepidea } \\
\text { amatymbica } \\
\text { Eckl. \& Zeyh. }\end{array}$ & Shrub & Bark/roots & $\begin{array}{l}\text { Aspergillosis, boost appetite, cryptococcal } \\
\text { meningitis, diarrhea, clean blood vessels, } \\
\text { oesopharyngeal candidiasis (South Africa) } \\
{[7,8]}\end{array}$ & $\begin{array}{l}\text { Antiretroviral activity } \\
{[19]}\end{array}$ \\
\hline $\begin{array}{l}\text { Daucus carota } \\
\text { L. } \\
\text { Apocynaceae }\end{array}$ & Herb & Leaves & Skin infections (Cameroon) [2] & $\begin{array}{l}\text { Inhibits HSV-1 } \\
\text { replication [20] }\end{array}$ \\
\hline $\begin{array}{l}\text { Cascabela } \\
\text { thevetia (L.) } \\
\text { Lippold }\end{array}$ & Shrub & Roots & Cough, tuberculosis (Uganda) [11] & $\begin{array}{l}\text { HIV-I reverse } \\
\text { transcriptase and } \\
\text { HIV-I intrase } \\
\text { inhibitor activities } \\
\text { [21] }\end{array}$ \\
\hline \multicolumn{5}{|l|}{ Asteraceae } \\
\hline $\begin{array}{l}\text { Artemisia afra } \\
\text { Jacq. ex Wild }\end{array}$ & Shrub & Leaves & $\begin{array}{l}\text { Oesopharyngeal candidiasis (South Africa) } \\
\text { [8] }\end{array}$ & Anti-HIV activity [22] \\
\hline $\begin{array}{l}\text { Artemisia annua } \\
\mathrm{L} \text {. }\end{array}$ & Shrub & Leaves & $\begin{array}{l}\text { Bacterial/fungal infections (Uganda) [11], } \\
\text { skin infections (Cameroon) [2] }\end{array}$ & Anti-HIV activity [22] \\
\hline $\begin{array}{l}\text { Aspilia pluriseta } \\
\text { Schweinf. }\end{array}$ & Herb & Leaves & Kwashiorkor, worms, wounds (Rwanda) [5] & Anti-HIV activity [5] \\
\hline $\begin{array}{l}\text { Tithonia } \\
\text { diversifolia } \\
\text { (Hemsl.) A. Gray }\end{array}$ & Shrub & Leaves & Ascariasis, diarrhea (Rwanda) [5] & Anti-HIV activity [5] \\
\hline $\begin{array}{l}\text { Vernonia } \\
\text { amygdalina Del. }\end{array}$ & Shrub & $\begin{array}{l}\text { Leaves/roo } \\
\text { ts }\end{array}$ & $\begin{array}{l}\text { Ascariasis, hepatitis, malaria (Rwanda) [5], } \\
\text { diarrhea, herpes zoster (Tanzania, Uganda) } \\
{[1,10,11] \text {, cryptococcal meningitis, herpes }} \\
\text { simplex (Cameroon, Tanzania) [1,2,10], skin } \\
\text { infections (Cameroon) [2], boost } \\
\text { appetite/immunity, stomach ache, fever } \\
\text { (Cameroon, Uganda) [2,11,13], skin rash } \\
\text { (Cameroon, Tanzania, Uganda) }[1,2,10,13] \text {, } \\
\text { backache, cough, headache (Cameroon, } \\
\text { Uganda) [2,13], stomachache (Uganda) [13] }\end{array}$ & Anti-HIV activity [23] \\
\hline $\begin{array}{l}\text { lia africana } \\
\text { n.) Benth. }\end{array}$ & Tree & Bark/fruits & $\begin{array}{l}\text { Herpes simplex (Namibia, Tanzania) } \\
{[1,3,4], \text { diarrhea (Namibia) [3], skin rash }} \\
\text { (Uganda) [12] }\end{array}$ & $\begin{array}{l}\text { Inhibits HIV-1 reverse } \\
\text { transcriptase [24] }\end{array}$ \\
\hline hanus sativus & Herb & Leaves & Skin infections (Cameroon) [2] & Antiviral activity [25] \\
\hline $\begin{array}{l}\text { ellaceae } \\
\text { burgia } \\
\text { idensis } \\
\text { gue } \\
\text { caceae }\end{array}$ & Tree & $\begin{array}{l}\text { Bark/leaves/r } \\
\text { oots }\end{array}$ & $\begin{array}{l}\text { Bacterial/fungal infection, cough, diarrhea, } \\
\text { tuberculosis (Uganda) [11] }\end{array}$ & $\begin{array}{l}\text { Inhibits HIV-1 reverse } \\
\text { transcriptase [24] }\end{array}$ \\
\hline ca papaya L. & Tree & $\begin{array}{l}\text { Leaves/roots } \\
\text { /seeds }\end{array}$ & $\begin{array}{l}\text { Cough, tuberculosis (Uganda) [11], oral } \\
\text { candidiasis (Tanzania) }[1,10]\end{array}$ & Anti- HIV-1 activity [26] \\
\hline
\end{tabular}




\begin{tabular}{|c|c|c|c|c|c|}
\hline \\
\hline $\begin{array}{l}\text { Celastraceae } \\
\text { Elaedendron } \\
\text { transvaalense (Burtt } \\
\text { Davy) R.H. Archer }\end{array}$ & Tree & \multicolumn{2}{|l|}{ Roots } & $\begin{array}{l}\text { Cold, dysmenorrhea, fever, skin rash } \\
\text { (South Africa) [6] }\end{array}$ & $\begin{array}{l}\text { Inhibits HIV-1 reverse } \\
\text { transcriptase [6] }\end{array}$ \\
\hline $\begin{array}{l}\text { Maytenus } \\
\text { senegalensis (Lam.) } \\
\text { Exell }\end{array}$ & Tree & \multicolumn{2}{|c|}{$\begin{array}{l}\text { Bark/leaves/r } \\
\text { oots }\end{array}$} & $\begin{array}{l}\text { Bacterial/fungal infections, cough (Uganda) } \\
\text { [11], herpes simplex/zoster, oral } \\
\text { candidiasis, skin rash, tuberculosis } \\
\text { (Uganda, Tanzania) }[1,10-12]\end{array}$ & $\begin{array}{l}\text { Inhibits HIV-1 } \\
\text { replication and } \\
\text { protease [24] }\end{array}$ \\
\hline \multicolumn{6}{|l|}{ Cleomaceae } \\
\hline $\begin{array}{l}\text { Cleome gynandra L. } \\
\text { Clusiaceae }\end{array}$ & Herb & \multicolumn{2}{|l|}{ Leaves } & Oral candidiasis (Tanzania) $[1,10]$ & Anti-HIV activity [27] \\
\hline $\begin{array}{l}\text { Garcinia buchananii } \\
\text { Bak. }\end{array}$ & Tree & \multicolumn{2}{|c|}{ Bark/roots } & $\begin{array}{l}\text { Cryptococcal meningitis, diarrhea, herpes } \\
\text { simplex/zoster, skin rash, tuberculosis } \\
\text { (Namibia, Tanzania) }[1,3,4]\end{array}$ & $\begin{array}{l}\text { HIV-1 protease } \\
\text { inhibitory activity [28] }\end{array}$ \\
\hline $\begin{array}{l}\text { Garcinia livingstonei } \\
\text { T. Anderson }\end{array}$ & Tree & \multicolumn{2}{|c|}{ Bark/roots } & $\begin{array}{l}\text { Cryptococcal meningitis, diarrhea, herpes } \\
\text { simplex/zoster, skin rash, tuberculosis } \\
\text { (Namibia) [3] }\end{array}$ & $\begin{array}{l}\text { HIV-1 protease } \\
\text { inhibitory activity [28] }\end{array}$ \\
\hline \multicolumn{6}{|l|}{ Combretaceae } \\
\hline $\begin{array}{l}\text { Combretum } \\
\text { hartmannianum } \\
\text { Schweinf. }\end{array}$ & Tree & \multicolumn{2}{|c|}{$\begin{array}{l}\text { Bark/leaves/ } \\
\text { stem }\end{array}$} & $\begin{array}{l}\text { Bacterial infections, febrile, jaundice } \\
\text { (Sudan) [9] }\end{array}$ & $\begin{array}{l}\text { Inhibits HIV-1 reverse } \\
\text { transcriptase [10] }\end{array}$ \\
\hline $\begin{array}{l}\text { Combretum molle } \\
\text { R. Br. ex G. Don }\end{array}$ & Tree & \multicolumn{2}{|c|}{ Bark/roots } & $\begin{array}{l}\text { Abdominal pains, convulsions, fever, } \\
\text { leprosy, snake bites, worms (South Africa) } \\
\text { [6], cough, tuberculosis (Uganda) [11] }\end{array}$ & $\begin{array}{l}\text { Inhibits HIV-1 reverse } \\
\text { transcriptase [6] }\end{array}$ \\
\hline $\begin{array}{l}\text { Combretum } \\
\text { paniculatum Vent. }\end{array}$ & Tree & \multicolumn{2}{|l|}{ Leaves } & Eye diseases, leprosy (Ethiopia) [29] & $\begin{array}{l}\text { Inhibits HIV-1 } \\
\text { replication [29] }\end{array}$ \\
\hline $\begin{array}{l}\text { Terminalia sericea } \\
\text { Burch. ex DC. } \\
\text { Cucurbitaceae }\end{array}$ & Tree & \multicolumn{2}{|l|}{ Bark } & Cryptococcal meningitis (Namibia) [4] & $\begin{array}{l}\text { Inhibits HIV-1 reverse } \\
\text { transcriptase [6] }\end{array}$ \\
\hline Cucurbita maxima & Climber & \multicolumn{2}{|l|}{ Leaves } & Skin infections (Cameroon) [2] & Anti-HIV activity [30] \\
\hline Cucurbita pepo L. & Climber & \multicolumn{2}{|c|}{ Leaves/roots } & Skin infections (Cameroon) [2] & $\begin{array}{l}\text { Inhibits HIV-1 reverse } \\
\text { transcriptase [31] }\end{array}$ \\
\hline $\begin{array}{l}\text { Momordica foetida } \\
\text { Schumach. }\end{array}$ & Herb & \multirow{2}{*}{\multicolumn{2}{|c|}{ Leaves }} & $\begin{array}{l}\text { Cough, diarrhea, fever, skin rash (Uganda) } \\
\text { [13] }\end{array}$ & Anti-HIV activity [32] \\
\hline \multicolumn{4}{|l|}{ Euphorbiaceae } & & \\
\hline $\begin{array}{l}\text { Croton lechleri Müll. } \\
\text { Arg. }\end{array}$ & Tree & Bark & \multicolumn{2}{|r|}{ Anaemia, boost appetite, diarrhea (Namibia) [3] } & Antiviral activity [33] \\
\hline Euphorbia hirta L. & Herb & $\begin{array}{l}\text { Leaves } / r \\
\text { oots }\end{array}$ & \multicolumn{2}{|r|}{$\begin{array}{l}\text { Bacterial/fungal infections, herpes zoster } \\
\text { (Uganda) [11], dermatophytoses (South Africa) [8] }\end{array}$} & $\begin{array}{l}\text { Inhibits HIV-1, } 2 \\
\text { reverse transcriptase } \\
\text { [34] }\end{array}$ \\
\hline Euphorbia tirucalli & Shrub & Leaves & \multicolumn{2}{|c|}{ Cough, tuberculosis (Uganda) [11] } & Anti-HIV activity [35] \\
\hline Jatropha curcas L. & Shrub & Leaves & \multicolumn{2}{|r|}{$\begin{array}{l}\text { Herpes zoster (Uganda) [11], oral candidiasis, skin } \\
\text { rash (Tanzania) }[1,10]\end{array}$} & Anti-HIV activity [36] \\
\hline $\begin{array}{l}\text { Ricinus communis } \\
\mathrm{L} \text {. }\end{array}$ & Shrub & Roots & \multicolumn{2}{|r|}{$\begin{array}{l}\text { Abortifacient, abscesses, antihelmentic, arthritis, } \\
\text { asthma, dermatitis, diarrhea, fever, flu, toothache, } \\
\text { tuberculosis, wounds (South Africa) [6], cough } \\
\text { (Tanzania) }[1,10]\end{array}$} & $\begin{array}{l}\text { Inhibits HIV-1 reverse } \\
\text { transcriptase [6] }\end{array}$ \\
\hline \multicolumn{6}{|l|}{ Fabaceae sensu lato } \\
\hline Abrus precatorius $\mathrm{L}$. & Climber & $\begin{array}{l}\text { Bark/leav } \\
\text { es/roots }\end{array}$ & \multicolumn{2}{|r|}{ Oral candidiasis (Namibia, Tanzania) $[1,3,10]$} & $\begin{array}{l}\text { Inhibits HIV-1 reverse } \\
\text { transcriptase [37] }\end{array}$ \\
\hline $\begin{array}{l}\text { Albizia amara } \\
\text { (Roxb.) B. Boiv. }\end{array}$ & Tree & Leaves & \multicolumn{2}{|c|}{ Stomach ache (Namibia) [3] } & $\begin{array}{l}\text { Inhibits HIV-1 reverse } \\
\text { transcriptase [38] }\end{array}$ \\
\hline $\begin{array}{l}\text { Cajanus cajan (L.) } \\
\text { Millsp. }\end{array}$ & Herb & Stem & \multicolumn{2}{|c|}{$\begin{array}{l}\text { Boost appetite/immunity (Uganda) [11], oral } \\
\text { candidiasis (Tanzania) }[1,10]\end{array}$} & Antiviral activity [39] \\
\hline $\begin{array}{l}\text { Dichrostachys } \\
\text { cinerea (L.) Wight \& } \\
\text { Arn }\end{array}$ & Shrub & Leaves & \multicolumn{2}{|c|}{ Oral candidiasis (Namibia) [3] } & $\begin{array}{l}\text { Inhibits HIV-1 reverse } \\
\text { transcriptase [16] }\end{array}$ \\
\hline $\begin{array}{l}\text { Erythrina abyssinica } \\
\text { DC. }\end{array}$ & Tree & Bark/root & \multirow{2}{*}{\multicolumn{2}{|c|}{$\begin{array}{l}\text { Cough, tubeculosis (Tanzania) [1,10], diarrhea, } \\
\text { herpes zoster (Uganda) [11] } \\
\text { Abdominal pains, cough, diarrhea, dysentery, } \\
\text { dysmenorrhea, infertility, sore throat, toothache, } \\
\text { tuberculosis, wounds (South Africa) [6] }\end{array}$}} & $\begin{array}{l}\text { Inhibits HIV-1 } \\
\text { replication [24] }\end{array}$ \\
\hline $\begin{array}{l}\text { Peltophorum } \\
\text { africanum Sond. }\end{array}$ & Tree & $\begin{array}{l}\text { Bark/root } \\
\mathrm{s}\end{array}$ & & & $\begin{array}{l}\text { Inhibits HIV-1 reverse } \\
\text { transcriptase [6] }\end{array}$ \\
\hline $\begin{array}{l}\text { Piliostigma } \\
\text { thonningii (Schum.) } \\
\text { Milne-Redh. }\end{array}$ & Tree & $\begin{array}{l}\text { Bark/root } \\
\text { s }\end{array}$ & \multicolumn{2}{|c|}{ Cough (Namibia) [4] } & $\begin{array}{l}\text { Anti-HIV, HSV-1 and } \\
2 \text { activity [40] }\end{array}$ \\
\hline
\end{tabular}




\begin{tabular}{|c|c|c|c|c|}
\hline $\begin{array}{l}\text { Senna alata (L.) } \\
\text { Roxb. }\end{array}$ & Tree & Leaves & Skin infections (Cameroon) [2] & $\begin{array}{l}\text { Inhibits HIV-1 reverse } \\
\text { transcriptase [37] }\end{array}$ \\
\hline $\begin{array}{l}\text { Senna occidentalis } \\
\text { (L.) Link }\end{array}$ & Herb & $\begin{array}{l}\text { Leaves } / r \\
\text { oots }\end{array}$ & $\begin{array}{l}\text { Cough (Namibia) }[3,4] \text {, diarrhea (Tanzania) }[1,10] \text {, } \\
\text { jaundice, malaria (Sudan) }[9]\end{array}$ & $\begin{array}{l}\text { Inhibits HIV-1 reverse } \\
\text { transcriptase [41] }\end{array}$ \\
\hline $\begin{array}{l}\text { Sutherlandia } \\
\text { frutescens } \mathrm{R} \text {. Br. }\end{array}$ & Shrub & $\begin{array}{l}\text { Leaves/t } \\
\text { wigs }\end{array}$ & $\begin{array}{l}\text { Backache, cold, chicken pox, diabetes, flu, } \\
\text { physical and mental stress, piles, rheumatism, } \\
\text { varicose veins (South Africa) [6] }\end{array}$ & $\begin{array}{l}\text { Inhibits HIV-1 reverse } \\
\text { transcriptase [6] }\end{array}$ \\
\hline
\end{tabular}

\section{Hypoxidaceae}

Hypoxis

hemerocallidea

(Fisch.) Mey. \&

Avé-Lall

Lamiaceae

Leonotis leonurus

(L.) R. Br.

Herb Tuber

Cancer, blood cleansing, boost appetite/immunity,

\section{Ocimum basilicum}

Herb

Herb

Leaves

Plectranthus

amboinicus

(Lour.)Spreng.

Plectranthus

barbatus Andr.

Malvaceae

Grewia mollis Juss.

\section{Meliaceae}

Azadirachta indica

A. Juss

\section{Melianthaceae}

Bersama abyssinica Tree

Boyle

Moringaceae

Moringa oleifera

Lam.

\section{Myricaceae}

Morella salicifolia

(Hochst. ex A.

Rich.) Verdc. \&

Polhil

\section{Myrisinaceae}

Maesa lanceolata

Forssk.

\section{Myrtaceae}

Eucalyptus globulus

Labil.

Psidium guajava L. Tree

Syzygium cordatum

Hochst. ex Krauss

Syzygium

guineense (Willd)

DC.

Olacaceae

Ximenia americana L.

\section{Oleaceae}

Olea europaea L. Tree
Tree Leaves Bacterial/fungal infections (Uganda) [11]

Bark/leav Ascariasis, cholera, diarrhea, dysentery, malaria, es/roots rabies, ulcers, worms (Ethiopia) [29]

Shrub

Leaves/s eeds/

Boost appetite/immunity (Uganda) [11], diarhea, skin infections, vomiting (Cameroon, Namibia) [2,3]

Tree

Bark/root

$\mathrm{s}$

Boost appetite/immunity (Uganda) [11], cryptococcal meningitis, diarrhea, herpes simplex, tuberculosis (Tanzania) $[1,10]$

Shrub Seeds

Leaves

Cough, tuberculosis (Uganda) [11]

Bark/leav Cough, diarrhea (Namibia, Uganda) [3,11-13], es tuberculosis (Namibia, Tanzania, Uganda) $[1,3,4,11]$, vaginal candidiasis (South Africa) [8]

Tree

Tree

Bark/leav

Diarrhea (Namibia)

es

Bark

Diarrhea (Namibia, Tanzania) $[1,3,10]$ oesopharyngeal candidiasis, tuberculosis, vaginal candidiasis, wounds (South Africa) $[7,8,17]$ wigs

Leaves

Inhibits HIV-1 reverse transcriptase [42]

Inhibits HIV-1

reverse transcriptase [16]

Inhibits HIV-1 reverse transcriptase [43] Anti-HIV, HSV-1 and 2 activity [44]

Anti-HIV-1 activity [45]

Inhibits HIV-1 replication and protease [24]

Inhibits HIV-1 replication and protease [24]

Inhibits HIV-1 replication [29]

Anti-HIV activity [46]

Inhibits HIV-1 replication and protease [24]

Inhibits HIV-1 replication [29]

Anti-HSV-1 and 2 activity [47] HIV-1 protease inhibitory activity [48] Anti-HIV activity [49] Inhibits HIV-1 reverse transcriptase [50]

Bark/root Contagious diseases, stomach ache, worms (Ethiopia) Inhibits HIV-1 [29], oral candidiasis (Namibia) [4], skin rash (Tanzania) $[1,10]$

replication [29]

Anti-HIV activity [51] 


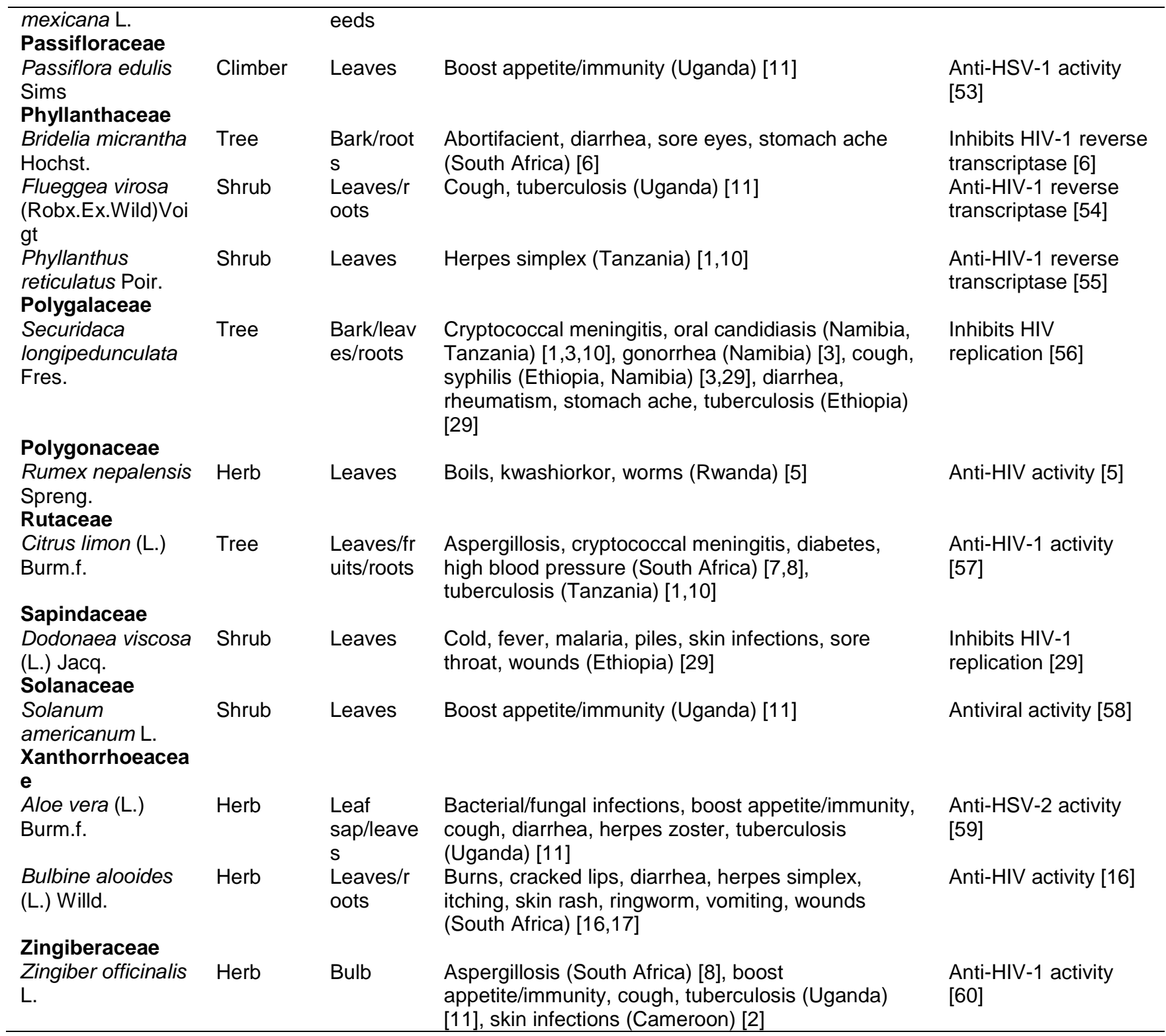

Table 2: Families with the largest number of medicinal plants (more than 2 species) used to treat and manage HIV/AIDS opportunistic diseases in subSaharan Africa

\begin{tabular}{lcc}
\hline Family & $\begin{array}{c}\text { Number of } \\
\text { medicinal plants }\end{array}$ & $\%$ \\
\hline $\begin{array}{l}\text { Fabaceae sensu } \\
\text { lato }\end{array}$ & 11 & 14.9 \\
\hline Asteraceae & 5 & 6.8 \\
\hline Combretaceae & 4 & 5.4 \\
Lamiaceae & 4 & 5.4 \\
Myrtaceae & 4 & 5.4 \\
Amaryllidaceae & 3 & 4.1 \\
Cucurbitaceae & 3 & 4.1 \\
Phyllanthaceae & 3 & 4.1 \\
Apiaceae & 2 & 2.7 \\
Celastraceae & 2 & 2.7 \\
Clusiaceae & 2 & 2.7 \\
Xanthorrhoeaceae & 2 & 2.7 \\
\hline
\end{tabular}

Table 3: Major ailment and disease categories and plant species reported. Most species were reported in more than one ailment and disease category.

\begin{tabular}{lc}
\hline Ailment/disease & $\begin{array}{c}\text { Number of } \\
\text { species }\end{array}$ \\
\hline Wounds & 8 \\
Bacterial/fungal infections & 9 \\
Fever & 9 \\
Boost immunity & 11 \\
Boost appetite & 13 \\
Cryptococcal meningitis & 13 \\
Oral/oesopharyngeal & 19 \\
candidiasis & \\
Herpes simplex/zoster & 27 \\
Tuberculosis & 30 \\
Skin infections/rash & 31 \\
Diarrhea & 32 \\
Cold/cough & 33 \\
\hline
\end{tabular}

Trop J Pharm Res, September 2014; 13(9): 1532 


\section{HIV/AIDS opportunistic diseases ailments treated with herbal medicines}

and

A total of 79 medical conditions related to HIV/AIDS were treated using herbal remedies (Table 1). Bacterial/fungal infections, boosting of appetite/immunity, cold/cough, cryptococcal meningitis, diarrhea, fever, herpes simplex/zoster, oral/oesopharyngeal candidiasis, skin infections/rash, tuberculosis and wounds were treated with the highest number of medicinal plant species (Table 3).

\section{DISCUSSION}

The checklist in Table 1 provides baseline data on medicinal plants that are currently used to treat and manage HIV/AIDS opportunistic diseases in sub-Saharan Africa. This inventory is a crucial starting point in trying to assess the efficacy of traditional medicines in the management of HIV/AIDS opportunistic diseases as well as the development of effective drugs for the treatment of HIV/AIDS. One of the possible approaches to finding novel anti-HIV therapeutic agents is the screening of medicinal plants that are widely used in local communities to treat and manage HIV/AIDS opportunistic diseases.

Out of 74 medicinal plants widely used in subSaharan Africa to treat and manage HIV/AIDS opportunistic diseases, 63 species $(85.1 \%$ ) have proven anti-HIV active compounds and known modes of action (Table 1). Among these is Kigelia africana used to treat diarrhea and herpes simplex in Namibia [3], herpes simplex in Tanzania [1] and skin rash in Uganda [12]. Maytenus senegalensis is used as herbal medicine for bacterial/fungal infections and cough in Uganda [11], and to treat herpes simplex/zoster, oral candidiasis, skin rash and tuberculosis in both Tanzania and Uganda [1,1012]. Similarly, Garcinia buchananii has the same ethnomedicinal applications in Namibia [3] and Tanzania [1] as herbal medicine for chronic diarrhea, cryptococcal meningitis, herpes simplex/zoster, skin rash and tuberculosis $[1,3,10]$.

Another noteworthy plant species used for treating and managing HIV/AIDS opportunistic diseases in sub-Saharan Africa are Hypoxis hemerocallidea and Sutherlandia frutescens (Table 1). These two species are currently recommended by the South African Ministry of Health for HIV management $[6,17,42]$. This is in accordance with the World Health Organization's recommendation that traditional medicines should be included in national responses to HIV/AIDS pandemic [14]. Some of these species can, therefore, be targeted for phytochemical and pharmacological studies with the aim of identifying active ingredients contained by such plants required for treating and managing HIV/AIDS. Such research is important, as there is need to search for improved antiretroviral agents which can be added to or replace the current drugs in use.

Despite the presence of anti-HIV activity in more than $85 \%$ of the plant species in Table 1, these plant species have not been fully explored. Further investigations on phytochemical constituents and subsequent screening are needed for opening new opportunities to develop pharmaceutical drugs based on anti-HIV constituents shown by some of these plant species.

This study revealed that many people in subSaharan Africa use traditional medicines for the treatment and management of HIV/AIDS opportunistic diseases despite the increasing availability of antiretroviral therapy. People in sub-Saharan Africa, particularly the poor are still dependent on medicinal plants for the treatment of basic human ailments like backache, cold, cough, diarrhea, fever, headache, skin infections and wounds $[14,17,24,44]$. Therefore, traditional medicines remain the main source of primary health care available and accessible to all people in sub-Saharan Africa.

Although no plant-derived drug is currently in clinical use to treat and manage HIV/AIDS opportunistic diseases, knowledge on these plants is very important as this can serve as leads in the discovery of new anti-HIV agents. Medicinal plants may yield novel compounds that may be of interest for HIV/AIDS drug development particularly those medicinal plants characterized by anti-HIV activity (Table 1).

\section{Limitations of the study}

This study has taken a focused approach and generated extensive exploratory and qualitative data, however, the following limitations are acknowledged in relation to its findings. Detailed accounts of plants used to treat and manage HIV/AIDS opportunistic diseases are available from Cameroon, Namibia, Rwanda, South Africa, Sudan, Tanzania and Uganda only. Although, the results from these countries are diverse in nature, they may not apply directly to all countries in the sub-Saharan Africa. The 
advantages of this limited sample size and sharp focus on detailed accounts on medicinal plants used to treat and manage HIV/AIDS opportunistic diseases in Cameroon, Namibia, Rwanda, South Africa, Sudan, Tanzania and Uganda resulted in a better and deeper understanding of traditional medicines useful for HIV/AIDS management in sub-Saharan Africa. Overall, while the study is useful in gaining an understanding of utilization of traditional medicines in managing HIV/AIDS opportunistic infections in sub-Saharan Africa and the value of traditional medicines in a wide spectrum of countries in the region, it is clear that more detailed national level studies should be undertaken. The present study provided a sample of preliminary data that could open up pertinent questions relating to the management of HIV/AIDS opportunistic infections using herbal medicines.

\section{CONCLUSION}

Correlation between the ethnomedicinal employment and the pharmacological activities have been duly observed and described in this study. In fact, this study suggests that some of the plant species have the potential to be developed as anti-HIV agents. While there are still gaps in the phytochemistry and pharmacological studies conducted so far, which need to be bridged in order to exploit the full medicinal potential of these species, it is still very clear that these plant species have tremendous potential for the future. This inventory will assist future workers on the selection of herbal plants to evaluate for phytochemical safety and pharmaceutical efficacy. There is also need for more research on the active compounds of these herbal medicines, some of which have already shown interesting pharmacological activities as shown in Table 1.

\section{REFERENCES}

1. Kisangau DP, Lyaruu HV, Hosea KM, Joseph CC. Use of traditional medicines in the management of HIVIAIDS opportunistic infections in Tanzania: a case in the Bukoba rural district. J Ethnobiol Ethnomed 2007; 3: 29.

2. Noumi N, Manga PN. Traditional medicines for HIV/AIDS and opportunistic infections in North-West Cameroon: case of skin infections. Amer J Trop Med Pub Health 2011; 1(3): 44-64.

3. Chinsembu KC, Hedimbi M. An ethnobotanical survey of plants used to manage HIV/AIDS opportunistic infections in Katima Mulilo, Caprivi region, Namibia. J Ethnobiol Ethnomed 2010; 6: 25.

4. Hedimbi M, Chinsembu KC. Ethnomedicinal study of plants used to manage HIVIAIDS-related disease conditions in the Ohangwena region, Namibia. Int $\mathrm{J}$ Med PI Res 2012; 1(1): 4-11.

5. Cos $P$, Hermans $N$, De Bruyne $T$, Apers $S$, Sindambiwe $J B$, Witvrouw M, De Clercq E, Van den Berghe $D$, Pieters L, Vlietinck AJ. Antiviral activity of Rwandan medicinal plants against human immunodeficiency virus type-1 (HIV-1). Phytomed 2002; 9: 62-68.

6. Bessong PO, Obi CL, Andréola M-L, Rojas LB, Pouységu L, Igumbor E, Meyer JJ, Quideau S, Litvak S. Evaluation of selected South African medicinal plants for inhibitory properties against human immunodeficiency virus type 1 reverse transcriptase and integrase. J Ethnopharmacol 2005; 99(1): 83-91.

7. Omoruyi BE, Bradley G, Afolayan AJ. Ethnomedicinal survey of medicinal plants used for the management of HIVIAIDS infection among local communities of Nkonkobe Municipality, Eastern Cape, South Africa. J Med PI Res 2012; 6(19): 3603-3608.

8. Otang WM, Grierson DS, Ndip R. Ethnobotanical survey of medicinal plants used in the management of opportunistic fungal infections in HIV/AIDS patients in the Amathole District of the Eastern Cape Province, South Africa. J Med PI Res 2012; 6(11): 2071-2080.

9. Ali $H$, Konig GM, Khalid SA, Wright AD, Kaminsky $R$. Evaluation of selected Sudanese medicinal plants for their in vitro activity against hemoflagellates, selected bacteria, HIV-1-RT and tyrosine kinase inhibitory and for cytotoxicity. J Ethnopharmacol 2002; 83(3): 219228.

10. Kisangau DP, Herrmann TM, Lyaruu HVM, Hosea KM, Joseph CC, Mbwambo ZH, Masimba PJ. Traditional knowledge, use practices and conservation of medicinal plants for HIVIAIDS care in rural Tanzania. Ethnobot Res Appl 2011; 9: 43-57.

11. Asiimwe S, Kamatenesi-Mugisha M, Namutebi A, BorgKarlsson A-K, Musiimenta P. Ethnobotanical study of nutri-medicinal plants used for the management of HIV/AIDS opportunistic ailments among the local communities of western Uganda. J Ethnopharmacol 2013; 150(2): 639-648.

12. Lamorde M, Tabuti JR, Obua C, Kukunda-Byobona C, Lanyero H, Byakika-Kibwika P, Bbosa GS, Lubenga A, Oqwal-Okeng J, Ryan M, Waako PJ, Merry C. Medicinal plants used by traditional medicine practitioners for the treatment of HIVIAIDS and related conditions in Uganda. J Ethnopharmacol 2010; 130(1): 43-53.

13. Langlois-Klassen D, Kipp W, Jhangri GS, Rubaale T. Use of traditional herbal medicine by AIDS patients in Kabarole District, Western Uganda. Am J Trop Med Hyg 2007; 77(4): 757-763.

14. Rates SMK. Plants as a source of drugs. Toxicon 2001; 39: 603-613. 
15. Silprasit $K$, Seetaha $S$, Pongsanarakul $P$, Hannongbua $S$, Choowongkomon K. Anti-HIV-1 reverse transcriptase activities of hexane extracts from some Asian medicinal plants. J Med PI Res 2011; 5(17): 41944201.

16. Klos M, van de Venter M, Milne PJ, Traore HN, Meyer D, Oosthuizen V. In vitro anti-HIV activity of five selected South African medicinal plant extracts. $J$ Ethnopharmacol 2009; 124(2): 182-188.

17. van Wyk BE, van Oudtshoom B, Gericke N. Medicinal plants of South Africa. Pretoria: Briza Publications; 2000. $336 \mathrm{p}$.

18. Shah KA, Patel MB, Patel RJ, Parmar PK. Mangifera Indica (Mango). Pharmacogn Rev 2010; 4(7): 42-48.

19. Louvel S, Moodley N, Seibert I, Steenkamp $P$, Nthambeleni $R$, Vidal V, Maharaj V, Klimkait $T$. Identification of compounds from the plant species Alepidea amatymbica active against HIV. South Afr J Bot 2013; 86: 9-14

20. Torky ZA. Antiviral activity of polyphenols extracts from Daucus carota against Herpes simplex virus type 1. TOJSAT: The Online J Sci Technol 2013; 3(1): 20-32.

21. Tewtrakul S, Nakamura $N$, Hattori M, Fujiwara $T$, Supavita T. Flavanone and flavonol glycosides from the leaves of Thevetia peruviana and their HIV-1 reverse transcriptase and HIV-1 integrase inhibitory activities. Chem Pharm Bull (Tokyo) 2002; 50(5): 630-635.

22. Lubbe A, Seibert I, Klimkait T, van der Kooy F. Ethnopharmacology in overdrive: the remarkable antiHIV activity of Artemisia annua. J Ethnopharmacol 2012; 141(3): 854-859.

23. Johnson CE, Lin L-Z, Harnly JM, Oladeinde FO, Kinyua $A M$, Michelin $R$, Bronner $Y$. Identification of the phenolic components of Vernonia amygdalina and Russelia equisetiformis. J Nat Prod 2011; 4: 57-64.

24. Rukanga GM, Kofi-Tsekpo MW, Kurokawa M, Kageyama S, Mungai GM, Muli JM, Tolo FM, Kibaya RM, Muthaura CN, Kanyara JN, Tukei PM, Shiraki K. Evaluation of the HIV-1 reverse transcriptase inhibitory properties of extracts from some medicinal plants in Kenya. Afr J Health Sci 2002; 9(1): 81-90.

25. Gutiérrez RMP, Perez RL. Raphanus sativus (Radish): Their chemistry and biology. The Sci World J 2004; 4 : 811-837.

26. Rashed $K$, Luo $M-T$, Zhang L-T, Zheng $Y-T$. Phytochemical screening of the polar extracts of Carica papaya Linn. and the evaluation of their antiHIV-1 activity. J Appl Industrial Sci 2013; 1(3): 49-53.

27. Mnzava NA, Chigumira Ngwerume F. Cleome gynandra L. In: Grubben GJH, Denton OA (eds). PROTA 2. Vegetables/Légumes. [CD-Rom]. PROTA Wageningen, Netherlands; 2003.

28. Magadula JJ, Tewtrakul S. Anti-HIV-1 protease activities of crude extracts of some Garcinia species growing in Tanzania. Afr J Biot 2010; 9(12): 1848-1852.

29. Asres K, Bucar F, Karting T, Witrouw M, Pannecouque C, Clercq ED. Antiviral activity against human immunodeficiency virus type 1 (HIV-1) and type 2 (HIV-2) of ethnobotanically selected Ethiopian medicinal plants. Phytother Res 2001; 15(1): 62-69.

30. Daly NL, Koltay A, Gustafson KR, Boyd MR, Casas-Finet $J R$, Craik DJ. Solution structure by NMR of circulin A: A macrocyclic knotted peptide having anti-HIV activity. J Mol Biol 1999; 285(1): 333-345.

31. Gerhäuser C, Samtleben R, Tau GT, Pezzuto JM, Lottspeich F, Wagner H. Peponin, a new ribosomeinactivating protein isolated from seeds of Curcubita pepo L. inhibits human immunodeficiency virus type 1 reverse transcriptase. Pharm Pharmacological Lett 1993; 3: 71-75.

32. Bourinbaiar AS., Lee-Huang S. Potentiation of anti-HIV activity of anti-inflammatory drugs, dexamethasone and indomethacin, by MAP30, the antiviral agent from bitter melon. Biochem Biophys Res. Commun 1995; 208(2): 779-885.

33. Williams JE. Review of Antiviral and immunomodulating properties of plants of the Peruvian rainforest with a particular emphasis on Uña de Gato and Sangre de Grado. Altern Medicine Rev 2001; 6(6): 567-579.

34. Gyuris A, Szlávik L, Minárovits J, Vasas A, Molnár J, Hohmann J. Antiviral activities of extracts of Euphorbia hirta L. against HIV-1, HIV-2 and SIVmac251. In Vivo 2009; 23(3): 429-432.

35. Upadhyay B, Singh KP, Kumar A. Ethno-medicinal, phytochemical and antimicrobial studies of Euphorbia tirucalli L. J Phytology 2010; 2(4): 65-77.

36. Kaur R, Kharb R. Anti-HIV potential of medicinally important plants. Int J Pharma Bio Sciences 2011; 2(3): 387-398.

37. Tewtrakul S, Subhadhirasakul S, Rattanasuwan P. HIV-1 protease inhibitory effects of some selected plants in Caesalpiniaceae and Papilionaceae families. Songklanakarin J Sci Technol 2003; 25(4): 509-514.

38. Mar W, Tan GT, Cordell GA, Pezzuto JM, Jurcic K, Offermann $F$, Redl $K$, Steinke $B$, Wagner $H$. Biological activity of novel macrocyclic alkaloids (budmunchiamines) from Albizia amara detected on the basis of interaction with DNA. J Nat Prod 1991; 54(6): 1531-1542.

39. Nwodo UU, Ngene AA, Iroegbu CU, Onyedikachi OAL, Chigor VN, Okoh Al. In vivo evaluation of the antiviral activity of Cajanus cajan on measles virus. Arch Virol 2011; 156: 1551-1557

40. Lemessa, D. Piliostigma thonningii (Schumach.) MilneRedh. In: Brink M, Achigan-Dako EG. (eds). Prota 16: Fibres/Plantes à fibres. [CD-Rom]. PROTA, Wageningen, Netherlands; 2010.

41. Lunavath $V$, Estari $M$. Inhibition of human immunodeficiency virus (HIV-1) reverse transcriptase by Cassia occidentalis L. plant extract. Int J Sci Engi Res 2012; 3(7): 1-4.

42. Klos M. In vitro anti-HIV and immunomodulatory activity of five selected South African medicinal plant extracts [dissertation]. [Port Elizabeth]: Nelson Mandela Metropolitan University; 2006. 184 p.

Trop J Pharm Res, September 2014; 13(9): 1535 
43. Yamasaki K, Nakano M, Kawahata $T$, Mori $H$, Otake $T$, Ueba $N$, Oishi I, Inami R, Yamane $M$, Nakamura $M$, Murata $H$, Nakanishi T. Anti-HIV-1 activity of herbs in Labiatae. Biol Pharm Bull 1998; 21(8): 829-833.

44. Williams CJ. Medicinal plants in Australia: An antipodean apothecary. Rosenberg Publishing, NSW, Australia; 2013

45. Kapewangolo $P$, Hussein AA, Meyer D. Inhibition of HIV1 enzymes, antioxidant and anti-inflammatory activities of Plectranthus barbatus. J Ethnopharmacol 2013; 149(1): 184-190.

46. Nworu C S, Okoye EL, Ezeifeka GO, Esimone CO. Extracts of Moringa oleifera Lam. showing inhibitory activity against early steps in the infectivity of HIV-1 lentiviral particles in a viral vector-based screening. Afr J Biotechnol 2013; 12(30): 4866-4873.

47. Schnitzler $P$, Schon $K$, Reichling J. Antiviral activity of Australian tea tree oil and eucalyptus oil against herpes simplex virus in cell culture. Pharmazie 2001; 56: 343-347.

48. Mao QC, Zhou YC, Li RM, Hu YP, Liu SW, Li XJ. Inhibition of HIV-1 mediated cell-cell fusion by saponin fraction from Psidium guajava leaf. Zhong Yao Cai 2010; 33: 1751-1754.

49. Hurinanthan V. Anti-HIV activity of selected South African medicinal plants [dissertation]. Durban University of Technology, Durban; 2013. $169 p$.

50. Moll A, Heyman HM, Meyer JJM. Plants with activity against the live $\mathrm{HI}$ virus and the enzyme, reverse transcriptase. South Afr J Bot 2013; 86: 148.

51. Qian Y, Guan T, Tang X, Huang L, Huang M, Li Y, Sun $H$. Maslinic acid, a natural triterpenoid compound from Olea europaea, protects cortical neurons against oxygen-glucose deprivation-induced injury. Eur J Pharmacol 2011; 670(1): 148-153.

52. Chang YC, Hsieh PW, Chang FR, Wu RR, Liaw CC, Lee $K H, W u$ YC. Two new protopines argemexicaines $A$ and $B$ and the anti-HIV alkaloid 6acetonyldihydrochelerythrine from formosan
Argemone mexicana. Planta Med 2003; 69(2): 148152.

53. Müller V, Chávez JH, Reginatto FH, Zucolotto SM, Niero $R$, Navarro D, Yunes RA, Schenkel EP, Barardi CRM, Zanetti CR, Simões CMO. Evaluation of antiviral activity of South American plant extracts against herpes simplex virus type 1 and rabies virus. Phytother Res 2007; 21(10): 970-974.

54. Wiwat C, Kwantrairat S. HIV- 1 reverse transcriptase inhibitors from Thai medicinal plants and Elephantopus scaber Linn. Mahidol University $J$ Pharm Sci 2013; 40(3): 35-44.

55. Eldeen IMS, Seow E-M, Abdullah R, Sulaiman SF. In vitro antibacterial, antioxidant, total phenolic contents and anti-HIV-1 reverse transcriptase activities of extracts of seven Phyllanthus sp. South Afr J Bot 2011; 77(1): 75-79

56. Muazu J, Kaita AH. A review of traditional plants used in the treatment of epilepsy amongst the Hausa/Fulani tribes of northern Nigeria. Afr J Trad Complement Altern Med 2008; 5(4): 387-390.

57. Lackman-Smith CS, Snyder BA, Marotte KM, Osterling MC, Mankowski MK, Jones M, Nieves-Duran L, Richardson-Harman N, Cummins JE, Sanders-Beer $B E$. Safety and anti-HIV assessments of natural vaginal cleansing products in an established topical microbicides in vitro testing algorithm. AIDS Res Therapy 2010; 7: 22.

58. Javed T, Ashfaq UA, Riaz S, Rehman S, Riazuddin S. Invitro antiviral activity of Solanum nigrum against Hepatitis C Virus. Virology Journal 2011; 8:26

59. Zandi K, Zadeh MA, Sartavi K, Rastian Z. 2007. Antiviral activity of Aloe vera against herpes simplex virus type 2: An in vitro study. Afr J Biotechn 2007; 6(15): 17701773.

60. Feng T, Su J, Ding ZH, Zheng YT, Li Y, Leng Y, Liu JK. Chemical constituents and their bioactivities of "Tongling White Ginger" (Zingiber officinale). J Agric Food Chem 2011; 59(21): 11690-11695. 